\title{
Electromagnetic Pulse Coupling Analysis of Electronic Equipment
}

Lei Hong ${ }^{1}$, LI Qingying ${ }^{2}$

${ }^{1}$ Aviation Industry Corporation of China, Shenyang Aircraft Design Institute, Shenyang, China

${ }^{2}$ Electronic Information Engineering, Shenyang Aerospace University, Shenyang, China

\begin{abstract}
High-intensity nuclear explosion caused by high-altitude nuclear electromagnetic pulse through the antenna, metal cables, holes and other channels, coupled with very high energy into the electronic device, and cause serious threats. In this paper, the mechanism, waveform, coupling path and damage effect of nuclear electromagnetic pulse is analyzed, and the coupling mechanism of nuclear electromagnetic pulse is studied.
\end{abstract}

Keywords Nuclear electromagnetic pulse Coupling Cable Hole seam

\section{Electromagnetic Pulse}

The electromagnetic pulse (EMP) has a short duration and a bandwidth. Electromagnetic pulse is usually caused by the explosion (especially nuclear explosion) and other conditions of electromagnetic radiation, acting on electronic materials, blasting equipment or the surrounding medium of electromagnetic shock wave. Any form of nuclear explosion can produce electromagnetic pulse, but the form is different, high altitude nuclear explosion caused by electromagnetic pulse greater damage, nuclear electromagnetic pulse intensity, high frequency components rich, covering a wide area. For more than $100 \mathrm{~km}$ high altitude nuclear explosion, the resulting high field strength in the ground coverage of up to thousands of kilometers. Therefore, this paper mainly discusses the high-altitude nuclear explosion electromagnetic pulse (HEMP). Electromagnetic radiation environment produced by high altitude nuclear explosion, early $(0<\mathrm{t}<1 \mu \mathrm{s})$, medium $(1 \mu \mathrm{s}<\mathrm{t}$ $<1 \mathrm{~s})$ and late ( $>1 \mathrm{~s})$ from time to time. Which early nuclear electromagnetic pulse on the destruction of electronic systems plays a major role. At present, there are many kinds of description of HEMP radiation environment at home and abroad, mostly in the form of double exponent as the mathematical expression of radiation waveform, the time domain expression is ${ }^{[1]}$ :

$$
E(t)=k E_{0}\left(e^{-\alpha t}-e^{-\beta t}\right)
$$

Where $\mathrm{k}$ is the correction coefficient, $E_{0}$ is the peak field strength, generally $50 \mathrm{kV} / \mathrm{m}, \beta, \alpha$ is the characterization of the pulse before and after the parameters. The figure1 shows the waveform of the electromagnetic pulse when the parameter is $k=1.3, E_{0}=50 \mathrm{kV} / \mathrm{m}, \alpha=4 \times 10^{7}, \beta=6 \times 10^{8}$.

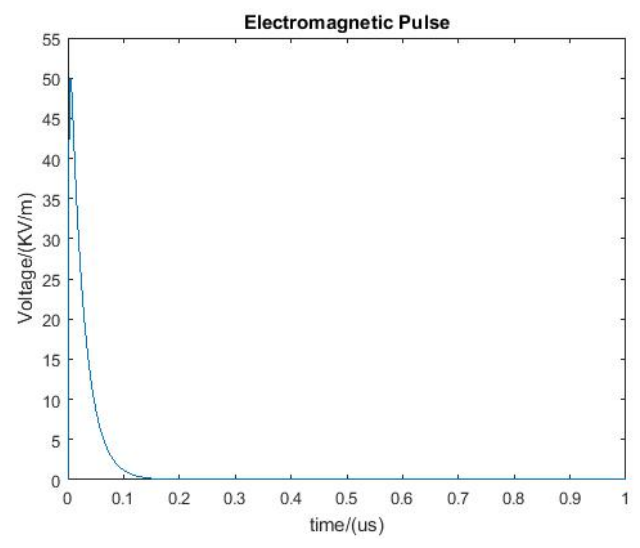

Figure 1 electromagnetic pulse waveform 


\section{Damage effect of electromagnetic pulse}

Electromagnetic pulse coupling into the electronic system or equipment will affect the electronic components, lines and the entire system or equipment, so that the bit error rate, electronic components performance degradation or even damage, resulting in communication system performance degradation or failure, known as electromagnetic pulse effect. This effect may be temporary or permanent. The sensitivity of the system is the response of the system to the transient electromagnetic shock of the coupled electromagnetic pulse. The system caused by the impact of electromagnetic pulse temporary work disorders called work failure, and permanent damage is called functional damage.

Electromagnetic pulse no obvious damage to humans and non-electronic devices. The damage caused by electromagnetic pulse is related to its field strength and pulse width, which is related to nuclear explosion equivalent, burst height and distance. The effect of electromagnetic pulses on electronic systems or devices can be divided into four levels, namely: ineffective, Interference, disturbance and damage, depending on the size of the coupling energy entering the target, the coupling energy representing the incoming electronic system or device and subsequently generating The power to enter the target electronics depends on the power that can be generated by the electromagnetic pulse, the distance from the target, and the characteristics (frequency, pulse rate, pulse duration, etc.) of the electromagnetic pulse radiation, as well as the protection capability of the target. Electromagnetic pulse mainly through the energy of the conduction coupling, radiation coupling mode, the damage mode can be summarized as the following four aspects:

\subsection{Thermal effect}

The thermal effect of electromagnetic pulses is usually done in nanoseconds or microseconds, and is an adiabatic process. This effect can be used as ignition source and detonating source, instantaneous cause flammable, explosive gas and other items burning explosion; Can also make the communication system in the microelectronic devices, electromagnetic sensitive circuit overheating or input protection resistance burned, resulting in local thermal damage, resulting in deterioration of circuit performance or failure, and even lead to inventory burning explosion.

\subsection{Interference and "surge" effects}

The transient over-voltages or "surge" effects of electromagnetic pulses can also cause hard damage to the communication system. The performance of the device or circuit may deteriorate or completely fail, mainly for the semiconductor device open circuit, short circuit, current gain drop, leakage current increases, metal melting, and oxide breakdown; May also form a cumulative effect, buried the potential harm, so that the reliability of the circuit or equipment to reduce, resulting in malfunction.

\subsection{Strong electric field effect}

The strong electric field formed by the electromagnetic pulse source can not only cause the dielectric breakdown between the gate oxide layer and the metallization line of the metal oxide semiconductor (MOS) circuit in the communication device, so that the circuit fails, and the system self-test instrument and the sensitive device.

\subsection{Magnetic effect}

The strong current caused by the electromagnetic pulse can produce a strong magnetic field, so that the electromagnetic 
energy directly coupled to the system, interfere with the normal work of the communication system.

\section{Coupling Path of Electromagnetic Pulse}

Electromagnetic pulses can be damaged by the front door coupling and back door coupling to the electronic system. Front door coupling: The communication system must transmit the baseband signal to the transmit antenna before it can be effectively propagated outward, and vice versa. When the communication system is irradiated with electromagnetic pulses (especially when the main valve is aligned), the pulse signal is received by the antenna and transmitted to the RF port. RF module contains a band-pass filter, the purpose is to select the useful signal, abandon the out-of-band signal. But the face of the electromagnetic pulse is too large amplitude, the ordinary band-pass filter simply cannot withstand, the risk of being burned out.

Backdoor Coupling: Electromagnetic pulses cause large transient currents or electrical standing waves through the fixed electrical connections of the device and the interconnected cables of the device, which can cause damage to the circuit inside the device. The back door coupling of the communication system is mainly through the power line, the signal line, the cavity hole coupling to the inside of the system ${ }^{[2]}$.

\subsection{Front door coupling}

Electromagnetic pulse coupling to the electronic device communication system transceiver antenna will produce the induced voltage or current, of course, but also consider the antenna band response and gain, the frequency is very high in a very short antenna can sense a large voltage. These voltages or currents are transmitted through the cable to the inside of the system to affect the normal operation of the system, which can damage the electronic circuits and their components in severe cases.

Electromagnetic pulse through the electronic equipment of the power line, the signal line input and output, coupled with electromagnetic pulse energy to produce a certain strength of the voltage or current signal, and then in a conductive way into the electronic device to go. Even if the current is not introduced from the leader of the transmission cable core, but in the outer shield on the induction, through the transfer impedance will be coupled to the core, directly into the electronic device system ${ }^{[3]}$.

There must be more than one module within a system; each module may not be under the same mechanical structure under the shield. The transmission of power, control and signal between different modules is mostly done by cable. Cables tend to be long and exposed to the air. When the electromagnetic pulse is irradiated, the cable receives the electromagnetic wave through the antenna effect, especially when the cable length and the wavelength of the electromagnetic wave can be compared, the coupling is particularly serious.

The coupling of the EMP to the cable is generally on the order of 100 degrees and increases with the length of the cable and the height of the cable, which increases as the load resistance decreases across the cable.

Antenna system is the main carrier of the transmission and reception of the electronic equipment. The electromagnetic pulse energy is easy to enter the communication system of the electronic equipment through the "front door" coupling of the antenna, thus destroying the sensitive devices inside the electronic equipment. The center-fed dipole antenna shown in Fig. 2 is taken as an example. 


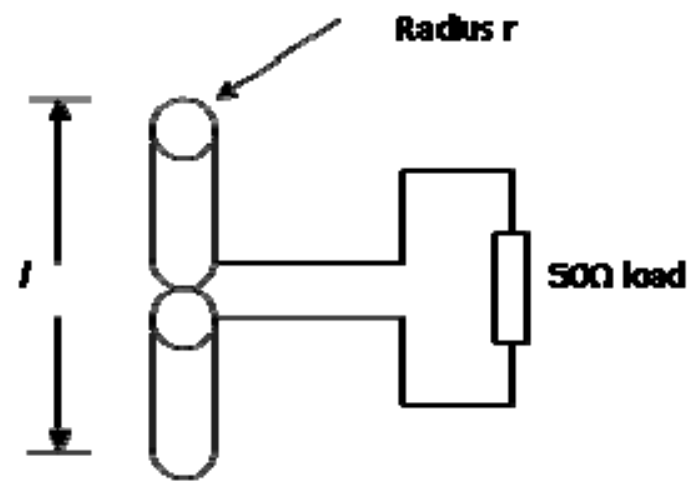

free 2 Doler loed dipole

Its structural parameters are:

$$
\chi=2 \ln \left(\frac{l}{r}\right)
$$

Where: $\chi$ for the antenna structure parameters, $l$ for the antenna length $(m), \mathrm{r}$ antenna radius $(m)$.

The resonant frequency of the antenna is:

$$
f_{c}=\frac{c}{2 l}
$$

Where: $f_{c}$ for the antenna resonant frequency $(\mathrm{Hz}), \mathrm{c}$ for the electromagnetic wave propagation velocity in vacuum $(\mathrm{m} / \mathrm{s}), l$ for the antenna length $(m)$.

$50 \Omega$ load on the flow through the transient induced current mathematical expression is:

$$
I_{L}(t)=k I_{p} e\left(\frac{-\pi f_{c} t}{Q}\right) \sin \left(2 \pi f_{c} t\right) \quad t \geq 0
$$

Where: $I_{L}(t)$ is the antenna load induced current (A) $I_{p}$ is the current peak (A), $Q$ is the damping coefficient.

The damping coefficient of the center feed dipole antenna $Q=\chi / 3.6$. According to, the maximum induced current of the antenna in free space is ${ }^{[4]}$ :

$$
I_{\max }=H l
$$

Where: $I_{\max }$ is the maximum induced current (A), $H$ is the incident wave magnetic field $(\mathrm{A} / m)$.

From the above formula, the induced current waveform for the attenuation of oscillation, the cycle is about $\frac{2 l}{c}$, where $l$ is the length of the wire. $c$ is the speed of light in free space. As the length of the wire increases, the magnitude of the induced current increases ${ }^{[5]}$.

\subsection{Backdoor coupling}

In order to facilitate the monitoring of the electronic system to open the ventilation system, in addition to the electronic chassis structure on the surface due to the existence of surface roughness, resulting in a random gap. These leaks provide coupling channels for electromagnetic pulses. When the size of the hole is greater than half the wavelength of the electromagnetic wave, the electromagnetic wave can enter the chassis, greatly reducing the shielding effectiveness of the chassis. Once the electromagnetic pulse into the box inside, bare Internet and printed circuit board can receive all kinds of electromagnetic pulse, into the sensitive circuit will damage the components, affecting the entire system. Take the seam as an example:

$$
V(\omega)=I(\omega) Z_{T}(\omega)
$$

Where $\omega$ is the electromagnetic wave angular frequency $(\mathrm{rad} / \mathrm{s}), V(\omega)$ is the seam's induced voltage $(V), I(\omega)$ is the current flowing through the joint $(\mathrm{A}), Z_{T}(\omega)$ is the transfer impedance of the joint $(\Omega)$.

The transfer impedance of the joint is related to the direction of the surface current. When the current is perpendicular to the joint, the transfer impedance is the largest, and when the surface current is parallel to the joint, the transfer impedance is very small ${ }^{[6] . ~ A c c o r d i n g ~ t o ~ t h e ~}$ literature, the coupling electric field perpendicular to the joint direction is larger than the coupling electric field parallel to the joint direction $^{[4]}$.

\section{Concluding remarks}

In summary, the coupling of electromagnetic pulse is mainly for front door coupling and backdoor coupling. The electromagnetic pulse enters into the interior of the electronic device by coupling and causes a degree of damage to it. To study how the electromagnetic pulse on the 
electronic equipment, electrical system damage, so as to study the corresponding protection methods, we must first clear the electromagnetic pulse coupling path. Different electromagnetic pulse coupling path due to different frequencies, coupling, the way is also different. Electromagnetic pulse through the front of the electronic equipment coupling or back door coupling into the system will cause serious damage to electronic equipment.

\section{References}

1. GAO Guangguang, GUO Xiaoqiang, ZHOU Hui, XIE Yanzhao. Double Exponential Function Fitting Method for Simulated Waveform of High-altitude Nuclear Electromagnetic Pulse [J]. Strong laser and particle beam. 2004, 14 (3): 336-340.

2. LU Xinke. Effect and Protection of Electromagnetic Pulse [D]. Xi'an: Xi'an University of Electronic Science and Technology master's degree thesis, 2009.

3. ZHANG Xin. Study on Coupling Simulation of Electromagnetic Pulse on Cable [D]. Xi'an: Xi'an University of Electronic Science and Technology master's degree, 2014.

4. Xiong Run. Study on Coupling of Electromagnetic Pulse on Equipment Chassis Lap Joint [J]. Modern Physics , 2015, 5, 88-92.

5. ZHU Lijie, SHEN Weidong, WANG Jianli, WU Honglin, GAO Guige, SHI Zhiqiang. Electrical pulse coupling and protection analysis of communication vehicle $[\mathrm{J}]$. Mobile power and vehicles. 2012, (3): $24-29$.

6. Xia Nenghong, Yi Xueqin, Song Wenwu, Huang Songgao. Study on Coupling Characteristics of Metal
Chassis with Punching under EMP

Excitation $\quad[\mathrm{J}]$ Equipment

Environmental Engineering. 2009, 6 (3): 21-24 